\title{
ATITUDES DOS CONSUMIDORES A PARTIR DA TEORIA DAS PISTAS E DA CONSCIÊNCIA AMBIENTAL: CONTRIBUIÇÕES AO ESTUDO DO GREEN MARKETING
}

\author{
Consumer attitudes from the theory of clues and \\ environmental awareness: Contributions to the study \\ of green marketing
}

Envio 20.10.09 / Aceite 01.12.09

\author{
Aline Nadalin Velter ${ }^{1}$ \\ Luciana Flores Battistella ${ }^{2}$ \\ Márcia Zampieri Grohmann ${ }^{3}$ \\ Aline Egres Castro ${ }^{4}$ \\ Vânia Flores Costa ${ }^{5}$ \\ Rolf Erdmann Hermann ${ }^{6}$
}

\footnotetext{
${ }^{1}$ Bacharel em Administração de Empresas pelo Centro Universitário Franciscano, MBA em Marketing pela Escola de Pós-Graduação em Economia da Fundação Getulio Vargas e mestranda em Administração da Universidade Federal de Santa Maria (UFSM). E-mail: aline.velter@gmail.com

${ }^{2}$ Bacharel em Administração pela UFSM, mestrado em Comunicação e Cultura pela Escola de Comunicação da Universidade Federal do Rio de Janeiro e doutorado em Engenharia de Produção pela Universidade Federal de Santa Catarina (UFSC). Professora Adjunta do Departamento de Ciências Administrativas da UFSM. E-mail: luttibattistella@ gmail.com

${ }^{3}$ Bacharel em Administração pela UFSM, mestrado em Engenharia de Produção pela UFSM e doutorado em Engenharia de Produção pela UFSC. Professora Adjunta do Departamento de Ciências Administrativas da UFSM. E-mail: marciazg@gmail.com

${ }^{4}$ Bacharel em Administração e mestranda em Administração, ambos pela UFSM. E-mail: alineeggres@gmail.com

${ }^{5}$ Bacharel em Administração pela UFSM, mestrado em Administração pela UFSC e doutorado em Administração pela Universidade Federal da Bahia (2008). Professora Adjunta do Departamento de Ciências Administrativas da UFSM. E-mail: vaniaflores2006@yahoo.com.br

${ }^{6}$ Bacharel em Administração e Engenharia Mecânica, mestrado em Engenharia de Produção (1984) e doutorado em Engenharia de Produção, todos pela UFSC. Professor Associado da UFSC. Pesquisador CNPq nível 2. E-mail: erdmann@cse.ufsc.br
} 


\title{
Resumo
}

Com o crescente interesse da sociedade por temas relacionados às questões ecológicas e ambientais, diversos estudos emergem com o objetivo de identificar o reflexo dessa preocupação socioambiental nos hábitos de consumo. Este estudo tem o objetivo de investigar o impacto de um argumento de caráter ecológico sobre atitudes relativas à marca, ao anúncio e à intenção de compra (LAGES e NETO, 2002; PEREIRA, 2003; ROCHA e MARQUES, 2004; BEDANTE e SLONGO, 2004; ROMEIRO, 2006; VEIGA, SANTOS e LACERDA, 2006; ENOKI et al., 2008; GARCIA et al., 2008; GIL e CAMARGO, 2008; PIMENTA e BOAS, 2008; BERTOLINI, POSSAMAI e BRANDALISE, 2009; MOTTA, 2009). Para tanto, foram reaplicados os modelos utilizados por Pereira (2003), a escala desenvolvida por Sujan (1985) e Sujan e Bettman (1989) para mensurar as atitudes dos consumidores em relação ao anúncio, marca e a sua intenção de compra; e a escala de Roberts (1996) e Straughan e Roberts (1999), traduzida para o português por Lages e Neto (2002), elaborada para medir a consciência ecológica dos consumidores. Os dados foram coletados, com 176 estudantes de graduação do curso de Administração de uma universidade do interior do Rio Grande do Sul, através de um estudo experimental do tipo between subjects, com três níveis de manipulação. As hipóteses previam que sujeitos que foram expostos ao argumento ecológico demonstram atitude mais favorável em relação à embalagem $(\mathrm{H} 1 \mathrm{~A})$, à marca (H1B), e intenção mais favorável de compra $(\mathrm{H} 1 \mathrm{C})$ que aqueles que não foram expostos ao argumento ecológico. Adicionalmente foi inserido no estudo o tratamento com o atributo intrínseco "Protege os dentes", com o objetivo de reforçar os resultados da pesquisa. Apesar da média das atitudes ser, na sua maioria, maior para o tratamento com o argumento ecológico, os resultados demonstraram que os respondentes mostraram-se indiferentes à presença de argumentos ecológicos sobre as atitudes em relação à embalagem, à marca e à intenção de compra. No entanto, constatou-se que a variável demográfica sexo e o hábito de compra dos respondentes interferem nas suas atitudes referentes à embalagem e à intenção de compra. Desta forma pode-se inferir, a partir deste estudo, que o argumento ecológico não é um grande influenciador no comportamento do consumidor. Também se verificou a consciência ecológica dos consumidores como influenciadora das atitudes em relação à embalagem, à marca e à intenção de compra. $O$ resultado com maior significância $(F=5,304$, Sig. $=0,022)$ mostrou que os estudantes que apresentam uma consciência ambiental acima da média demonstram uma baixa intenção de compra.

Palavras-chave: Teoria das Pistas; Consciência Ambiental; Comportamento do Consumidor.

\begin{abstract}
With the growing interest of society for issues related to ecological and environmental issues, several studies have emerged in order to identify the reflection of socio and environmental concern in consumer habits. This study aims to investigate the impact of an argument of ecological character in attitudes towards brand, ad and purchase intention (LAGES \& NETO, 2002; PEREIRA, 2003; ROCHA \& MARQUES, 2004, 2004; BEDANTE and SLONG, 2004; PALMER, 2006; VEIGA, SANTOS \& LACERDA, 2006; ENOKI et al., 2008; GARCIA et al., 2008; GIL \& CAMARGO, 2008; \& HOT PEPPER, 2008; BERTOLINI, POSSAMAI \& BRANDALISE, 2009; MOTTA, 2009). To do so, was reapplied the models used by Pereira (2003), the scale developed by Sujan (1985) and Sujan \& Bettman (1989) to measure consumer attitudes towards the advertising, brand and intention to purchase, and the scale of Roberts (1996) and Straughan \& Roberts (1999), translated to Portuguese by Lages \& Neto (2002), designed to measure the environmental awareness of consumers. The data were collected, with 176 undergraduate students of the course of administration of a university in Rio Grande do Sul, through an experimental study of the type "between subjects" with three levels of manipulation. The hypothesis predicted that subjects who were exposed to the ecological clue demonstrate more favorable attitude in relation to the packaging (H1A), brand $(H 1 B)$ and more favorable purchase intention (H1C) than those who were not exposed to the ecological. Additionally, was included in the study the treatment with the intrinsic attribute "Protects the teeth" in order to enhance the search results. Although the average attitude is, mostly, higher for treatment with the ecological argument, the results showed that respondents tended to be indifferent to ecological arguments about attitudes towards packaging, brand and purchase intention. However, it appeared that
\end{abstract}

Rev. Adm. UFSM, Santa Maria, v. 2, n. 3, p. 399-416, set./dez. 2009 
the demographic variable gender and respondents' purchasing habits interfere in their attitudes regarding the packaging and purchase intent. Thus it can be inferred from this study that the ecological argument is not a big influencer of consumer behavior. Also there was the ecological awareness of consumers as influential in attitudes in relation to packaging, brand and purchase intention. The result with greater significance $(F=5.304$, Sig. $=0.022)$ showed that students who have an environmental conscience above average show a lower purchase intent.

Keywords: Clue Theory; Environmental Awareness; Consumer Behavior.

\section{Introdução}

Com o aumento dos diversos meios de comunicação de massa, nos últimos anos, a exposição dos consumidores à informação passou a ser maior. Dessa forma, há uma tendência para elevar o nível de consciência e sensibilidade dos consumidores às causas ambientais (DIAS, 2008). Segundo Portilho (1999), a partir do crescimento das pressões humanas sobre a degradação do meio ambiente e a interferência direta que este tem na qualidade de vida das pessoas, cresce o chamado movimento ambientalista. Engajado a esse movimento, o consumidor ecológico manifesta suas preocupações ambientais nas suas atitudes e, consequentemente, no seu comportamento de compra. Esse consumidor busca produtos que causam um menor impacto ao meio ambiente e valorizam os produtos produzidos por empresas ambientalmente responsáveis (DIAS, 2008).

De acordo com Fishbein e Ajzen (1975, p. 216), a atitude é definida como um "sentimento positivo ou negativo de um indivíduo com relação a um determinado objeto de comportamento". Os consumidores têm atitudes em relação a uma ampla gama de objetos: desde comportamentos muito específicos relacionados a produtos (como comprar um creme dental de uma marca e não de outra) até comportamentos mais gerais relativos ao consumo (como comprar em uma loja da maneira tradicional ou através de comércio eletrônico).

A partir desses fatos, surge a importância do argumento ecológico, o qual é definido por Pereira (2003) como uma informação de caráter ecológico presente na embalagem do produto. Ao se deparar com o argumento ecológico presente em uma embalagem, espera-se que o consumidor avalie positivamente a embalagem e a marca, e, com isso, a intenção de compra seja favorável (MINIARD, BHATLA e ROSE, 1990; LUTZ, MACKENZIE e BELCH, 1983; MACKENZIE, LUTZ e BELCH, 1986).

Os problemas de deteriorização do meio ambiente e a busca por um consumo socialmente responsável têm sido alvo de pesquisas tanto pelo meio acadêmico quanto pela iniciativa empresarial (ASHLEY, 2002). Pode-se citar o trabalho de Pickett-Backer e Ozaki (2008), o qual buscou identificar as influências do marketing verde na decisão de compra de produtos pró-ambientais do consumidor. Já o estudo de Kreidler e Joseph-Mathews (2009) introduziu a ideia de "atmosfera verde" e uma tipologia para os consumidores verdes. Lin (2009) averiguou os consumidores atuais e potenciais de algodão orgânico no Havaí.

No Brasil, alguns estudos acadêmicos já se dedicaram à questão ambiental, ora buscando uma visão de como as empresas estão agindo em relação ao meio ambiente (DINATO, 1999; BORGES, 2008; FABRIS, 2008, SCHULTZ-PEREIRA e GUIMARÃES, 2009), ora buscando verificar a visão e a sensibilidade dos consumidores sobre esta questão (LAGES e NETO, 2002; PEREIRA, 2003; ROCHA e MARQUES, 2004; BEDANTE e SLONGO, 2004; ROMEIRO, 
2006; VEIGA, SANTOS e LACERDA, 2006; ENOKI et al., 2008; GARCIA et al., 2008; GIL e CAMARGO, 2008; PIMENTA e BOAS, 2008; BERTOLINI, POSSAMAI e BRANDALISE, 2009; MOTTA, 2009).

Com o propósito de enriquecer a discussão sobre o comportamento do consumidor perante produtos com apelos ecológicos em suas embalagens e/ou propagandas, este estudo teve por objetivo examinar a reação dos consumidores à presença ou ausência do argumento ecológico e, desta maneira, proporcionar uma melhor compreensão sobre as atitudes dos consumidores em relação ao impacto de apelos ecológicos na propaganda. Para tanto, foram reaplicados os modelos utilizados por Pereira (2003). Esse autor investigou as atitudes dos consumidores em relação ao anúncio, marca e a sua intenção de compra, tendo por base a escala desenvolvida por Sujan (1985) e Sujan e Bettman (1989); também mediu a consciência ecológica de sua amostra utilizando a escala de Roberts (1996) e Straughan e Roberts (1999), traduzida para o português por Lages e Neto (2002). O presente estudo também buscou identificar as relações entre as atitudes relativas à embalagem, à marca e à intenção de compra; verificar as relações entre a intenção de compra e as atitudes em relação à marca e à embalagem; e analisar se a consciência ecológica do consumidor interfere nas suas atitudes e na intenção de compra.

Este trabalho será dividido em dois estudos: as atitudes e a teoria das pistas (estudo 1) e a consciência ambiental e as atitudes (estudo 2). Antes de abordar os dois estudos, o presente trabalho apresenta a introdução e os tópicos: atitudes e intenções comportamentais do consumidor e as atitudes em relação ao anúncio, à marca e à intenção de compra. O estudo 1 apresenta uma revisão da literatura sobre os atributos do produto e o paradigma das pistas e a metodologia utilizada. O estudo 2 estrutura-se em: consciência ecológica e método utilizado. Ao final são apresentados os resultados e as considerações finais.

\section{Atitudes e intenções comportamentais do consumidor}

O estudo das atitudes constitui um objetivo primordial para a compreensão da intenção do comportamento, pois é um determinante importante da mesma. Conforme Blackwell, Engel e Miniard (2008), o comportamento do consumidor engloba as atividades diretamente envolvidas em obter, consumir e dispor serviços, incluindo os processos decisórios que antecedem e sucedem essas ações. Já as intenções são definidas por Mowen e Minor (1998) como julgamentos subjetivos sobre como deverá ser o comportamento no futuro em relação à aquisição, disposição e uso de produtos e serviços. As intenções de consumo representam a predisposição de engajar-se em determinada atividade relacionada ao consumo (BLACKWELL, ENGEL e MINIARD, 2008).

De acordo com Fishbein e Ajzen (1975), a principal característica que distingue as atitudes de outros conceitos é a sua natureza avaliativa ou afetiva. As atitudes são desenvolvidas pelos consumidores sobre quase tudo que os rodeiam, criando, assim, constatações positivas ou negativas, as quais os levam a comportar-se de forma coerente (KOTLER e KELLER, 2006).

No modelo de componentes definido por Schiffman e Kanuk (2000), as atitudes dividem-se em três componentes básicos: cognitivo, afetivo e conativo (comportamental). O primeiro consiste na elaboração (pensamentos) e o consequente conhecimento adquirido através de uma combinação da experiência com as informações disponíveis. Para Schiffman e Kanuk (2000), esse conhecimento e as percepções resultantes podem tomar a forma de crenças, isto é, o consumidor acredita que o objeto de atitude possui vários atributos. O componente afetivo refere-se às emoções ou sentimentos do consumidor quanto a um produto 
ou marca, gerados a partir da experiência afetiva da situação. Finalmente o componente conativo ou comportamental está relacionado com a probabilidade ou tendência do indivíduo em comportar-se de uma maneira específica. De acordo com Assael (1998), o elo entre esses três componentes ilustra o alto envolvimento hierárquico de efeitos: a confiança na marca influencia a avaliação da marca, que influencia a intenção de comprar.

Segundo Schiffman e Kanuk (2000), a formação de atitudes é facilitada pela experiência pessoal direta, sendo influenciada pelas ideias e experiências dos amigos, dos membros da família e pela exposição à comunicação de massa. Dessa forma, as questões ambientais são preponderantes para a formação da atitude do consumidor.

\subsection{A influência das atitudes em relação ao anúncio $\left(A_{\text {ant }}\right)$}

A atitude em relação ao anúncio $\left(A_{a n}\right)$ é definida como uma predisposição por parte do consumidor em responder, de forma favorável ou desfavorável, a um determinado estímulo de comunicação (LUTZ, 1991; SOLOMON, 2002). De acordo com Blackwell, Engel e Miniard (2008), a eficiência da propaganda em criar atitudes positivas em relação a um produto ou marca vai depender, , geralmente, das atitudes dos consumidores em relação ao próprio anúncio. Determinantes da $\mathrm{A}_{\mathrm{an}}$ incluem vários fatores, tais como: o anunciante, a qualidade do anúncio, o estado de espírito evocado pelo anúncio e os níveis de envolvimento do consumidor (BLACKWELL, ENGEL e MINIARD, 2008).

Conforme Pereira (2003), os sentimentos transmitidos por um anúncio não só podem influenciar as atitudes em relação ao anúncio em si, como também podem afetar as atitudes em relação à marca; logo um consumidor exposto a um anúncio ou embalagem de um produto pode demonstrar uma atitude neutra em relação ao anúncio, mas se sentir envolvido por algum apelo contido, como, por exemplo, um apelo ecológico na mensagem, poderá, desta maneira, apresentar uma melhor avaliação em relação à marca.

A $A_{a n}$ é uma importante conexão na cadeia causal entre as percepções dos atributos de um lado e as intenções e comportamentos de outro (LUTZ, 1991). Além das atitudes em relação aos atributos do produto e/ou serviço, é importante entender as atitudes em relação a outros tipos de associação com a marca. Essas associações podem incluir logomarcas, símbolos e celebridades que endossam produtos. Segundo Blackwell, Engel e Miniard (2008), um estudo realizado pela Advertising Research Foundation indicou que a opinião dos espectadores sobre um comercial de TV é um importante previsor do sucesso da eficiência do anúncio no mercado. Deste modo, a $\mathrm{A}_{\mathrm{an}}$ é uma importante variável a ser estudada na comunicação de marketing.

\subsection{A influência das atitudes em relação à marca $\left(A_{m}\right)$}

Antes de efetuar uma compra, o consumidor analisa os itens mais variados, desde a qualidade, custo, design, preço e marca. As marcas têm um peso primordial na tomada de decisão de compra, pois permite adquirir o produto com mais confiança. Quando o consumidor identifica os atributos de uma determinada marca, através da propaganda boca a boca ou pela experiência com ela, cada atributo torna-se conectado àquela marca em maior ou menor grau. Dessa forma, pode-se inferir que a atitude em relação a uma determinada marca $\left(A_{m}\right)$ é derivada das atitudes em relação aos atributos e benefícios desta marca (LUTZ, 1991).

Sendo a atitude em relação à marca uma avaliação por parte do consumidor, o sucesso de programas de marketing vai depender de associações favoráveis com a marca. Segundo Hoeffler e Keller (2002), programas de sucesso de marketing socioambiental corporativo têm 
construído vantagem competitiva para a empresa através do melhoramento de sua imagem corporativa frente aos consumidores e, consequentemente, melhorado a avaliação da marca.

Compreender a atitude do consumidor em relação à marca tem sido o foco de interesse de várias pesquisas de marketing (MITCHELL e OLSON, 1981; MINIARD, BHATLA e ROSE, 1990; LUTZ, MACKENZIE e BELCH, 1983). A maioria dessas pesquisas tem tentado compreender que as reações dos consumidores a um anúncio, na atitude em relação à marca, é mediada pela atitude em relação ao próprio anúncio (PEREIRA, 2003).

\subsection{Atitude em relação à intenção de compra $\left(A_{i c}\right)$}

As atitudes favoráveis em relação ao objeto atitude não se traduzem automaticamente em uma intenção de compra (BLACKWELL, ENGEL e MINIARD, 2008; FISHBEIN e AJZEN, 2005). Um consumidor pode gostar de uma marca, mas ter a intenção de comprar o produto de outra marca, pois aprecia este último mais do que o primeiro. Por essa razão, as atitudes são, algumas vezes, medidas na forma de preferências. As preferências representam as atitudes sobre um objeto em relação a outro (BLACKWELL, ENGEL e MINIARD, 2008). Ter uma atitude favorável em relação a um produto não é o mesmo que ter uma atitude favorável em relação a comprar ou consumir o produto (BLACKWELL, ENGEL e MINIARD, 2008). Ou seja, pode-se gostar de um determinado produto e/ou marca, mas preferir adquirir-se outra que possua um preço menor.

A maioria dos pesquisadores concorda que a $A_{a n}$ e a $A_{m}$ levam à intenção de compra (Aic) (LUTZ, 1991; AJZEN e FISHBEIN, 2005), sendo que essa relação permite usá-las como medidas previsoras do comportamento em relação a um objeto (Figura 1).

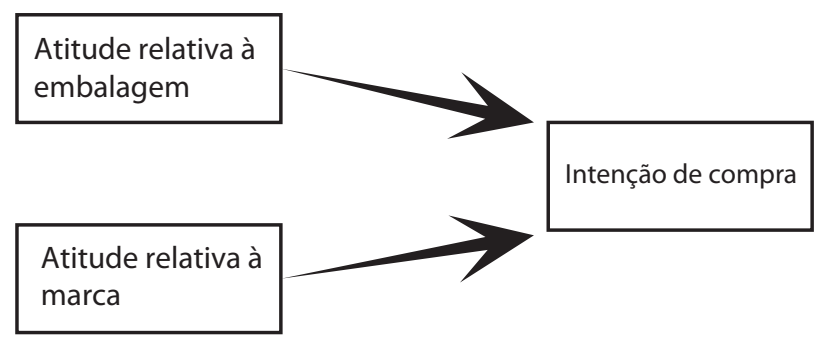

Fonte: Elaborada pelos autores

Figura 1 - Relação atitutes e intenção de compra.

\section{Estudo 1: As atitudes e a teoria das pistas}

Este estudo investiga o impacto de uma pista, no caso deste estudo, um argumento ecológico, nas atitudes dos consumidores em relação à embalagem, marca e intenção de compra. Segundo Olson e Jacoby (1972), produtos e marcas possuem uma gama de pistas (preço, nome da marca, embalagem, cor etc.). Estas pistas fornecem uma base para o desenvolvimento de várias impressões sobre o produto, incluindo a qualidade percebida, sendo esta fortemente relacionada com o real comportamento de compra do consumidor (OLSON e JACOBY, 1972).

\subsection{Atributos dos produtos e o paradigma das pistas}

Segundo Keller (1993), atributos são aquelas características que descrevem propriamente um produto ou serviço, o qual um consumidor avalia ao comprar um produto. 
Os atributos presentes na embalagem do produto ou em anúncios podem ser utilizados por consumidores para avaliar o produto, a marca ou a empresa. Esta avaliação relativa ao produto, marca ou empresa pode afetar as atitudes e o comportamento de compra.

Algumas teorias procuram entender como estas atitudes são formadas, enquanto outras se preocupam com mudança de atitudes, isto é, uma forma de persuadir os consumidores (BLACKWELL, ENGEL e MINIARD, 2008; SCHIFFMAN e KANUK, 2000). O atributo de um produto é o principal estímulo que influencia o consumidor em sua tomada de decisão de compra, avaliando-os em função de seus próprios valores, crenças ou experiências passadas (PETER e OLSON, 1996).

De acordo com Szybilo e Jacoby (1974), para que um ato de compra se concretize, os consumidores, em geral, realizam uma avaliação e julgamento comparativo entre produtos e marcas. Esse processo de avaliação passa pelo julgamento do que chamam de pistas associadas aos produtos ou serviços. Essas pistas são identificadas, avaliadas e integradas para compor um julgamento. As pistas seriam entendidas então como os vários itens de informações sugestivas sobre o produto, marca ou serviço que são identificadas, avaliadas e interpretadas para compor uma avaliação. Pistas com apelos ecológicos dariam sugestões sobre a forma ecologicamente correta com que determinado produto foi produzido.

De acordo com Olson e Jacoby (1972), estas pistas podem ser intrínsecas ou extrínsecas ao produto. As pistas intrínsecas são aquelas que podem ser modificadas ou manipuladas sem modificar as características físicas do produto, como sabor e ingredientes em um alimento, por exemplo. As pistas extrínsecas são aquelas relacionadas ao produto, mas que não fazem parte fisicamente deste, ou seja, que não estão diretamente relacionadas às características do produto e que podem ser alteradas sem que o produto se modifique.

Informações disponíveis relativas aos produtos devem gerar respostas (atitudes e comportamento) dos consumidores. Tendo por base o argumento presente na literatura popular de gestão (pop-management), argumentos ecológicos levam a atitudes favoráveis e a preferência por parte do consumidor (OTTMAN, 1994).

\subsection{Hipóteses}

Considerando que um argumento ecológico éveiculado em um estímulo mercadológico, ele pode afetar atitudes relativas à embalagem (neste estudo), à marca e à ideia de comprar o produto (intenção de compra) (MINIARD, BATHALA e ROSE, 1990; LORD, LEE e SAUER, 1995; NEWELL, GOLDSMITH e BANZHAF, 1998; MACKENZIE, LUTZ e BELCH, 1986). Dessa forma, as hipóteses formuladas são:

a) Hipótese $\mathrm{H}_{1}$ : comparando com os estudantes que receberam informações neutras, os estudantes que receberam informações com argumento ecológico:

$-\mathrm{H}_{1 \mathrm{~A}}$ : demonstram atitude mais favorável em relação à embalagem;

$-\mathrm{H}_{1 \mathrm{~B}}$ : demonstram atitude mais favorável em relação à marca;

$-\mathrm{H}_{1 \mathrm{C}}$ : demonstram atitude mais favorável em relação à intenção de compra.

Existe a possibilidade de os respondentes reagirem à presença de outro atributo qualquer e não especificamente ao argumento ecológico. Sendo o argumento ecológico considerado neste estudo um atributo extrínseco do produto, o atributo intrínseco do produto é chamado de "protege os dentes" com o objetivo de verificar se os estudantes da pesquisa reagem à presença especificamente do argumento ecológico ou não. Este atributo também teve

${ }^{3}$ www.hermespardini.com.br 
como objetivo comparar o impacto de atributos intrínsecos versus extrínsecos sobre as variáveis dependentes do estudo. Sendo assim, as seguintes hipóteses foram formuladas:

b) Hipótese $\mathrm{H}_{2}$ : comparando com os estudantes que receberam informações com o atributo "protege os dentes", os estudantes que receberam informações com o argumento ecológico:

$-\mathrm{H}_{2 \mathrm{~A}}$ : demonstram atitude mais favorável em relação à embalagem;

$-\mathrm{H}_{2 \mathrm{~B}}$ : demonstram atitude mais favorável em relação à marca;

$-\mathrm{H}_{2 \mathrm{C}}$ : demonstram atitude mais favorável em relação à intenção de compra.

\subsection{Metodologia}

Esta pesquisa se caracteriza como causal, pois pretende medir o efeito da presença de um argumento ecológico sobre atitudes relativas à embalagem, à marca e à intenção de compra. Para esse tipo de relação entre variáveis, o desenho escolhido mais apropriado é o experimental (PEDHAZUR e SCHMELKIN, 1991). A população para análise foi composta por estudantes de graduação do curso de Administração de uma universidade do interior do Rio Grande do Sul. Foi adotado um desenho experimental between subjects, ou seja, cada um dos grupos experimentais foi submetido a um tratamento.

Os respondentes foram aleatoriamente divididos em três grupos, os quais foram expostos fundamentalmente à mesma imagem. No primeiro grupo, a imagem ostenta o argumento ecológico; no segundo grupo, a imagem ostenta o atributo "protege os dentes"; e o terceiro grupo foi exposto à mesma embalagem sem o tratamento (tratamento 3 ou grupo de controle). Os três tratamentos podem ser visualizados na Figura 2.

O atributo "protege os dentes" diz respeito ao produto exposto não causar cáries, pois este não contém açúcar. Já o argumento ecológico utilizado nesta pesquisa foi o símbolo da reciclagem, passando a mensagem de que a embalagem utiliza materiais reciclados. A embalagem usada existe no mercado, é intensamente distribuída, e ostenta uma marca conhecida de chiclete. O argumento ecológico utilizado no experimento não está presente na embalagem real, comercializada da mesma forma que está exposta na figura utilizada na pesquisa. O selo ecológico foi colocado na embalagem com a ajuda de um programa de manipulação gráfica. Já o atributo intrínseco "não causa cáries" está presente na embalagem original do produto.

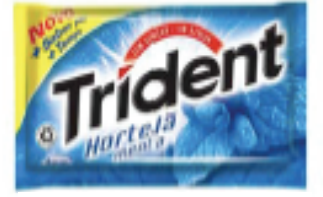

Tratamento com argumento ecológico

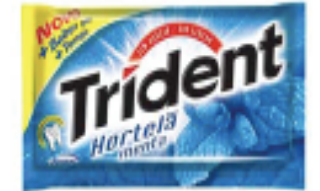

Tratamento com atributo "protege os dentes"

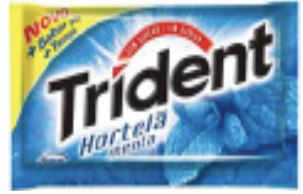

Embalagem sem tratamento

Fonte: Elaborada pelos autores

Figura 2 - Tratamentos utilizados na pesquisa.

Conforme procedimento utilizado por Sujan (1985) e Sujan e Bettman (1989), os respondentes indicaram suas atitudes em três escalas de cinco pontos: positiva/negativa; boa/ ruim; favorável/desfavorável para cada um dos três construtos: atitudes relativas à embalagem, à marca, e intenção de compra. 
Para Malhotra (2001), as variáveis dependentes são aquelas que medem o efeito das variáveis independentes sobre as unidades de teste, desse modo, há três variáveis dependentes: atitude em relação ao anúncio $\left(A_{a n}\right)$, atitude em relação à marca $\left(A_{m}\right)$ e atitude em relação à intenção de compra $\left(\mathrm{A}_{\mathrm{ic}}\right)$.

No caso desta pesquisa, este experimento foi conduzido para analisar a reação dos sujeitos em relação a uma embalagem de um produto com três níveis de manipulação (presença de argumento ecológico, presença de "protege os dentes" e ausência de argumento ecológico). O produto usado no experimento foi o chiclete Trident.

Depois de examinar a embalagem, os estudantes responderam um questionário com as medidas das variáveis dependentes e independentes. Este questionário foi elaborado para medir as atitudes dos consumidores em relação à categoria de produto, ao anúncio (embalagem), à marca e à intenção de compra.

\section{Estudo 2: A consciência ambiental e as atitudes}

O estudo 2 examina a consciência ambiental como um antecedente às atitudes em relação à embalagem, marca e intenção de compra. Conforme Bansal e Roth (2000), a preocupação de uma pessoa com o meio ambiente reflete em seus valores e influencia suas ações e atitudes.

\section{1 Consciência ambiental}

Com a proliferação de questões ecológicas nos meios de comunicação em massa e na Internet, observa-se uma contribuição à consciência ambiental da sociedade. Para Dias (2008), há uma tendência ao aumento do interesse pela proteção do meio ambiente, à medida que se agravam os problemas ambientais do planeta, como, por exemplo, a escassez de alguns recursos fundamentais (petróleo, água e energia). Schlegelmilch, Bohlen e Diamantopoulos (1996) definem consciência ambiental como um construto multidimensional que é composto por elementos cognitivos, atitudinais e comportamentais. Conforme Cuperschmid e Tavares (2001), o consumidor ambientalmente preocupado procura adotar atitudes e comportamentos de compra coerentes com a conservação dos ecossistemas.

Calzada (1998) define o consumidor ecologicamente correto como conscientizado e bem informado ambientalmente. Em suas compras, presta atenção e cuidado, olhando o rótulo do produto, verificando se possui algum selo de certificação, buscando informações como as da origem do produto, a forma como foi produzido e se possui garantia. De acordo com os estudos de Shrum, McCarty e Lowrey (1995), os consumidores verdes são formadores de opinião e têm um comportamento de compra cuidadoso à procura informações nos produtos.

Dessa forma, se os consumidores estiverem interessados na preservação do meio ambiente, tendo uma necessidade de cuidar da natureza, que pode ser reflexo de uma conscientização ambiental, eles se tornarão consumidores ecologicamente corretos e perceberão a oferta ou estímulo de empresas que produzem produtos ecológicos (BERTOLINI, POSSAMAI e BRANDALISE, 2009). Follows e Jobber (2000) afirmam que a consciência ecológica não é fator determinante para um comportamento de compra ecologicamente responsável. A intenção de compra do consumidor consciente ecologicamente é resultado de uma avaliação de tradeoff entre as consequências ambientais e as consequências individuais da compra. Ou seja, as consequências individuais podem explicar por que alguns consumidores com alta consciência ecológica não manifestarem comportamento de compra pró-meio-ambiente. 
Newell, Goldsmith e Banzhaf (1998) pesquisaram se consumidores expostos a anúncios contendo apelos ambientais com informações enganosas têm atitudes diferentes daqueles expostos a anúncios com apelos ambientais não enganosos. Esta falta de confiança em pistas com argumentos ecológicos pode causar a rejeição dessas pistas por parte dos consumidores (JOHANSSON, 1989).

\subsection{Metodologia}

Indo ao encontro dos procedimentos adotados no estudo 1, nesta etapa, a pesquisa também se caracteriza como causal, pois pretende medir o efeito da consciência ecológica sobre atitudes relativas à embalagem, marca e intenção de compra. A amostra utilizada é a mesma do estudo 1 e também foram utilizados os mesmos procedimentos no que se refere às variáveis dependentes: atitude em relação ao anúncio $\left(A_{a n}\right)$, atitude em relação à marca $\left(A_{m}\right)$ e atitude em relação à intenção de compra $\left(A_{i c}\right)$.

Porém, neste segundo estudo, a variável independente passa a ser a Consciência Ecológica. As variáveis independentes são aquelas "manipuladas pelo pesquisador e cujos efeitos são medidos e comparados" (MALHOTRA, 2001, p. 211).

A consciência ecológica será medida através da escala traduzida e validade por Lages e Neto (2002), a partir do modelo desenvolvido por Straugham e Roberts (1999). A escala original é composta por 30 itens e utiliza uma escala do tipo Likert de cinco pontos ( $1=$ nunca verdadeiro e 5 = sempre verdadeiro). Porém, seguindo os procedimentos de Pereira (2003), serão mensuradas quatro das seis dimensões da escala de Straugham e Roberts (1999), a saber, "produto", "reciclagem e reutilização", "alimentos" e "ação de mudança". Ou seja, serão excluídos os construtos "consumo de energia" e "hábitos domésticos".

Lages e Neto (2002) testaram a confiabilidade da escala e constataram que a maioria das dimensões obteve coeficientes bastante satisfatórios, e que a escala, como um todo, obteve um alfa de 0,8963. Neste estudo, a confiabilidade da escala, através do cálculo do alpha de Cronbach, foi superior a do estudo anterior, fornecendo um valor de 0,913 para os 18 itens analisados. Este valor é considerado muito bom e atesta a validade interna e confiabilidade da escala.

\section{Resultados}

A análise dos dados do presente estudo ficou dividida em cinco grupos: perfil da amostra, estatísticas descritivas, influências das variáveis demográficas, resultados do estudo 1 , resultados do estudo 2 .

\subsection{Perfil da amostra}

A amostra foi composta por 176 pessoas, das quais 88 são homens $(49,7 \%)$ e 89 são mulheres (50,3\%). Em relação à idade dos respondentes, 19\% possuem 19 anos; 49,7\% possuem até 20 anos; 39,5\% entre 20 a 25 anos; 7,3\% entre 25 a 30 anos e 3,4\% dos entrevistados têm mais de 30 anos. A maioria dos respondentes (52\%) apenas estuda e 47,5\% estudam e trabalham. Por fim, a amostra caracteriza-se por $53,7 \%$ de pessoas solteiras que moram com os pais ou com parentes, $37 \%$ que moram com amigos, $6 \%$ que moram com companheiros e $3 \%$ com companheiros e filhos.

Foi utilizado o Critério de Classificação Econômica Brasil de 2008, que assume que a classe social é função do poder de compra de uma lista de itens como geladeiras, carro, TV etc. 
Os resultados indicaram um perfil que se enquadra, na sua maioria, na classe A2 $(26,7 \%)$ e na Classe B1 (26,1\%). Os demais resultados são: 9\% classe A1; 23,3\% classe B2; 12,5\% classe C1 e $2,3 \%$ classe $\mathrm{C} 2$.

\subsection{Estatísticas descritivas}

Os entrevistados foram questionados sobre seus hábitos de compra, e os resultados encontrados foram de que a maioria absoluta $(92,7 \%)$ frequenta supermercados para comprar produtos alimentícios.

Apesar de o perfil dos entrevistados ser favorável para a compra de chiclete (produto escolhido neste experimento), os mesmos foram indagados sobre este tipo específico de produto e constatou-se que 65\% têm o hábito de comprar chiclete e, destes, 79,7\% adquirem especificamente a marca Trident, ou seja, a amostra escolhida tem frequente contato com o produto e o adquire com frequência.

Por fim, 37,6\% dos respondentes afirmaram que compram exatamente o chiclete mostrado anteriormente. Buscando compreender melhor se os entrevistados identificaram as pistas incluídas nas embalagens modificadas (Tratamento 1 e Tratamento 2), foi realizado o cruzamento das informações sobre consumir a marca e consumir exatamente este produto, utilizando-se como filtro a variável tratamento (Tabela 1). A partir da distribuição normal, o esperado é 22 e 37 no primeiro tratamento, entretanto o que foi obtido ou observado foi 24 e 35. A partir daí, vê-se se há diferença significativa.

Tabela 1 - Consumir a marca vs. consumir o produto

\begin{tabular}{|c|c|c|c|c|}
\hline & & \multicolumn{2}{|c|}{ Compra } & \multirow{2}{*}{ Total } \\
\hline & & $\operatorname{Sim}$ & Não & \\
\hline \multirow{2}{*}{ Argumento ecológico (tratamento 1) } & Observado & 24 & 35 & 59 \\
\hline & Esperado & 22,0 & 37,0 & 59,0 \\
\hline \multirow{2}{*}{ Protege os dentes (tratamento 2) } & Observado & 22 & 38 & 60 \\
\hline & Esperado & 22,4 & 37,6 & 60,0 \\
\hline \multirow{2}{*}{ Embalagem original (tratamento 3) } & Observado & 20 & 38 & 58 \\
\hline & Esperado & 21,6 & 36,4 & 58,0 \\
\hline \multirow{2}{*}{ Total } & Observado & 66 & 111 & 177 \\
\hline & Esperado & 66,0 & 111,0 & 177,0 \\
\hline
\end{tabular}

Fonte: Elaborada pelos autores.

Também foi analisada a média das atitudes em relação à embalagem, à marca e à intenção de compra (Tabela 2). A atitude que indicou maior média foi em relação à embalagem $(7,0366)$, a média total das atitudes (em relação à embalagem, marca e intenção de compra) foi de 5,1860. Com este resultado, percebe-se que a embalagem do produto tanto com o argumento ecológico quanto com o atributo "protege os dentes" e a sem nenhum tratamento causaram uma boa impressão aos respondentes. 
Tabela 2 - Média geral das atitudes

\begin{tabular}{lcc}
\hline & Média & Desvio-padrão \\
\hline Embalagem & 7,0366 & 2,80641 \\
Marca & 5,4624 & 2,17679 \\
Intenção compra & 4,5581 & 2,14179 \\
Atitude Total & 5,1860 & 1,82332 \\
\hline
\end{tabular}

Fonte: Elaborada pelos autores.

\subsection{Influências das variáveis demográficas}

Em relação à influência das variáveis demográficas nas atitudes, observa-se que a variável sexo influencia na intenção de compra do consumidor pesquisado (Tabela 3), sendo o público masculino o que possui maior pré-disposição para comprar produtos que apresentam argumentos ecológicos. Com este resultado, pode-se inferir que os consumidores do sexo masculino estão se sensibilizando mais com questões ambientais.

Tabela 3 - Influência da variável sexo nas atitudes

\begin{tabular}{lllccc}
\hline & SEXO & Média & Desvio-padrão & F & Sig. \\
\hline Intenção compra & Feminino & 4,2143 & 4,2143 & & \\
& Masculino & 4,8864 & 4,8864 & 5,088 & 0,025 \\
\hline
\end{tabular}

Fonte: Elaborada pelos autores.

Além da variável sexo, a outra variável que exerce nfluência sobre as atitudes é o hábito de compra (Tabela 4). A média das atitudes em relação à embalagem dos respondentes que não compram o produto é maior daqueles que compram. O mesmo comportamento apresentase também em relação à intenção de compra. Dessa forma, observa-se que os respondentes não compram o produto apresentaram uma atitude mais positiva em relação à embalagem e à intenção de compra.

Tabela 4 - Influência do hábito de compra nas atitudes

\begin{tabular}{llcccc}
\hline & SEXO & Média & Desvio-padrão & F & Sig. \\
\hline Embalagem & Compra & 6,0577 & 2,24615 & & \\
& Não compra & 8,7333 & 2,88695 & 6,696 & 0,011 \\
\hline \multirow{2}{*}{ Intenção compra } & Compra & 4,0000 & 1,54803 & & \\
& Não compra & 5,6000 & 2,66299 & 24,507 & 0,000 \\
\hline
\end{tabular}

Fonte: Elaborada pelos autores.

\subsection{Resultados do estudo 1}

As hipóteses $\left(\mathrm{H}_{1 \mathrm{~A}^{\prime}}, \mathrm{H}_{1 \mathrm{~B}}\right.$ e $\left.\mathrm{H}_{1 \mathrm{C}}\right)$ propõem que os estudantes que foram expostos ao argumento ecológico demonstram atitude mais favorável em relação à embalagem $\left(H_{1 A}\right)$, à 
marca $\left(\mathrm{H}_{1 \mathrm{~B}}\right)$ e à intenção de compra mais favorável $\left(\mathrm{H}_{1 \mathrm{C}}\right)$, que aqueles que foram expostos à imagem sem nenhum tratamento. Já as hipóteses $\left(\mathrm{H}_{2 \mathrm{~A}^{\prime}} \mathrm{H}_{2 \mathrm{~B}^{\prime}} \mathrm{H}_{2 \mathrm{C}}\right)$ colocam que os estudantes expostos ao argumento ecológico demonstram atitude mais favorável em relação à embalagem $\left(\mathrm{H}_{2 \mathrm{~A}}\right)$, à marca $\left(\mathrm{H}_{2 \mathrm{~B}}\right)$ e à intenção de compra mais favorável $\left(\mathrm{H}_{2 \mathrm{C}}\right)$ que aqueles expostos a imagem com atributo "protege os dentes".

Com o objetivo de testar essas hipóteses, foi conduzida uma análise das médias das variáveis dependentes do estudo em cada tratamento e os referidos desvios-padrão, os quais são apresentados na Tabela 5. A hipótese $\mathrm{H}_{1 \mathrm{~A}}$ afirma que, comparando com os sujeitos que receberam informações neutras, os sujeitos que receberam informações com argumento ecológico demonstram atitudes mais favoráveis em relação à embalagem. Os resultados mostram que há uma diferença pequena entre as médias da atitude em relação à embalagem entre o tratamentos 1 e o sem tratamento, sendo a média das atitudes sem o tratamento maior do que do tratamento 1 ; logo aceita-se a hipótese nula e a hipótese $\mathrm{H}_{1 \mathrm{~A}}$ não é confirmada. Conclui-se que os respondentes expostos ao argumento ecológico não demonstraram atitudes significativamente mais favoráveis em relação à embalagem do que os do grupo que não foi exposto ao argumento ecológico. Tal como o comportamento da $\mathrm{H}_{1 \mathrm{~A}^{\prime}}$ as $\mathrm{H}_{1 \mathrm{~B}}$ e $\mathrm{H}_{1 \mathrm{C}}$ também não são aceitas. Apesar da média das atitudes ser maior para o tratamento 1, não há uma diferença significante entre as médias do tratamento 1 e do sem tratamento e, dessa forma, aceita-se a hipótese nula e as $\mathrm{H}_{1 B}$ e $\mathrm{H}_{1 C}$ não são confirmadas. As hipóteses $\mathrm{H}_{2 A^{\prime}} \mathrm{H}_{2 B}$ e $\mathrm{H}_{2 C}$ também não são confirmadas, apesar de as médias das atitudes em relação à embalagem, à marca e à intenção de compra do tratamento 1 serem maiores do que as médias do tratamento 2, não há uma diferença significante entre as médias das atitudes.

Tabela 5 - Influência do tratamento nas atitudes

\begin{tabular}{llrrrr}
\hline & SEXO & Média & Desvio-padrão & F & Sig. \\
\hline Embalagem & Tratamento 1 & 7,0556 & 2,50597 & & \\
& Tratamento 2 & 6,8545 & 3,00258 & & \\
& Sem tratamento & 7,2000 & 2,92119 & 0,208 & 0,812 \\
\hline Marca & Tratamento 1 & 5,5789 & 2,09547 & & \\
& Tratamento 2 & 5,5593 & 2,45140 & & \\
& Sem tratamento & 5,2456 & 1,96651 & 0,420 & 0,658 \\
\hline Intenção compra & Tratamento 1 & 4,8947 & 2,48340 & & \\
& Tratamento 2 & 4,4138 & 2,03508 & & \\
& Sem tratamento & 4,3684 & 1,85772 & 1,060 & 0,349 \\
\hline
\end{tabular}

Fonte: Elaborada pelos autores.

\subsection{Resultados do estudo 2}

Para a análise desses resultados, foi utilizado o modelo de consciência ambiental, adaptado por Lages e Neto (2002). Para a identificação do nível final da consciência ecológica dos entrevistados, foi realizado o cálculo da média das médias obtidas nos quatro construtos analisados (produto, embalagem, reciclagem e saúde). Dessa forma, chegou-se a uma média geral de consciência ecológica. Os entrevistados foram classificados em dois grupos: aqueles acima da média e aqueles abaixo da média (destaca-se que os respondentes que ficaram dentro do intervalo de erro de $5 \%$ da média geral - nível de confiança de 95\%, não foram 
enquadrados nesta etapa do estudo). Como se observa na Tabela 6, o valor abaixo da média $(4,6875)$, da média da consciência ecológica da amostra, foi maior do que o valor acima da média $(4,3947)$. O resultado com maior significância $(F=5,304$, Sig. $=0,022)$ mostrou que os estudantes que apresentam uma consciência ambiental acima da média demonstram uma baixa intenção de compra.

Tabela 6 - Influência do consciência ambiental nas atitudes

\begin{tabular}{llcccc}
\hline & & Média & Desvio-padrão & F & Sig. \\
\hline \multirow{2}{*}{ Intenção compra } & Alta & 4,3947 & 1,77448 & & \\
& Baixa & 4,6875 & 2,39435 & 5,304 & 0,022 \\
\hline
\end{tabular}

Fonte: Elaborada pelos autores.

\section{Considerações finais}

No presente estudo, observou-se, comparando-se as atitudes dos consumidores que receberam a imagem com argumento ecológico aos dos que receberam a imagem sem nenhum tratamento, que há pouca diferença na média das atitudes em relação à embalagem, à marca e à intenção de compra. Assim, as hipóteses 1A, 1B e 1C não foram confirmadas, pois a diferença da média da atitude entre o tratamento 1 e o sem tratamento foi de pouca significância.

Esse resultado vai ao encontro dos estudos de Shrum, McCarty e Lowrey (1995), Lages e Neto (2002) e Pereira (2003), que sugerem que não é possível afirmar que a presença de apelos ecológicos afeta a intenção de compra do consumidor, e sim que estes apelos podem potencializar a relação entre a atitude em relação à marca e a intenção de compra do consumidor.

As hipóteses 2A, 2B e 2C também não são confirmadas. Este resultado é consistente com os estudos de Shrum, McCarty e Lowrey (1995) e Newell, Golsmith e Banzhaf (1998), pois os sujeitos da pesquisa parecem não ter avaliado melhor a embalagem com o argumento ecológico. Isto pode ter-se dado em razão de os consumidores se apresentarem céticos à embalagem em si e não ao argumento ecológico especificamente.

Outra explicação para essa aparente indiferença dos consumidores em relação às embalagens que contenham apelos ecológicos pode ser a falta de informação dos consumidores em geral. O grau de confiança do consumidor sobre a veracidade das informações contidas em pistas com argumento ecológico afetará a sua avaliação sobre estas pistas (OLSON e JACOBY, 1972).

Este resultado parece sugerir que os consumidores necessitam de mais informações sobre selos ecológicos presentes em produtos. A falta de clareza e de informações adicionais pode afetar a capacidade de avaliação e confiança do consumidor sobre as informações de determinada embalagem ou produto.

Quando analisados os dados referentes à consciência ecológica e sua influência nas atitudes (embalagem, marca e intenção de compra), verificou-se que os consumidores com consciência ambiental acima da média apresentaram baixa intenção de comprar o produto. Com este resultado, pode-se inferir que uma alta consciência ecológica não garante o comportamento de compra do consumidor. 


\section{Referências}

ASHLEY, P. A. Tendências do "dever-ser" quanto ao comércio ético de alimentos na gestão estratégica de supermercados a partir da práxis coletiva pesquisador-ASSERJ-ABRAS. 2002, 266 f. Tese (Doutorado em Administração) - Departamento de Administração - Pontifícia Universidade Católica do Rio de Janeiro, Rio de Janeiro, 2002.

ASSAEL, H. Consumer behavior and marketing action. 6. ed. Boston: PWS-KENT, 1998.

BANSAL, P.; ROTH, K. Why companies go green: a model of ecological responsiveness. The Academy of Management Journal, v. 43, n. 4, p. 717-736, 2000.

BEDANTE, G. N.; SLONGO, L. A. O comportamento de consumo sustentável e suas relações com a consciência ambiental e a intenção de compra de produtos ecologicamente embalado. In: ENCONTRO DE MARKETING DA ASSOCIAÇÃO NACIONAL DE PÓS-GRADUAÇÃO E PESQUISA EM ADMINISTRAÇÃO, EMA, 1., 2004, Porto Alegre. Anais... Porto Alegre: ANPAD, 2004. p. 1-16.

BERTOLINI, G. R. F; POSSAMAI, O.; BRANDALISE, L. T. A percepção dos consumidores de produtos ecologicamente corretos: estudo de caso em pequena empresa.

Revista da Micro e Pequena Empresa, v. 3, n. 1, p. 99-119, 2009.

BLACKWELL, R. D.; ENGEL, J. F.; MINIARD, P. W. Comportamento do Consumidor. 9. ed. Rio de Janeiro: Cengage Learning, 2008.

BORGES, M. S. Modelo de avaliação da estratégia ambiental: o estudo das vantagens competitivas na indústria cervejeira fluminense. In: SEMINÁRIOS EM ADMINISTRAÇÃO, SEMEAD, 11., 2008, São Paulo. Anais... São Paulo: SEMEAD, 2008. p. 1-12.

CALZADA, M. Quem é o consumidor orgânico. In: INTERNATIONAL FEDERATION OF
ORGANIC AGRICULTURE MOVEMENTS, IFOAM, Mar del Plata, 1998. Disponível em: $<$ http://www.planetaorganico.com.br/saudnovc. htm>. Acesso em: 17 nov. 2009.

CUPERSCHMID, N. R. M.; TAVARES, M. C. Atitudes em relação ao meio ambiente e sua influência no processo de compra de alimentos. In: ENCONTRO DA ASSOCIAÇÃO NACIONAL DE PÓS-GRADUAÇÃO E PESQUISA EM ADMINISTRAÇÃO, ENANPAD, 25., 2001, Campinas. Anais... Campinas: ANPAD, 2001. p. $1-11$.

DIAS, R. Marketing ambiental: ética, responsabilidade social e competitividade nos negócios. São Paulo: Atlas, 2008.

DINATO, M. R. O meio-ambiente e o setor petroquímico do Rio Grande do Sul: um estudo exploratório. 1999. 127f. (Mestrado em Administração) - Programa de Pós-Graduação em Administração - Universidade Federal do Rio Grande do Sul, Porto Alegre, 1999.

ENOKI, P. A. et al. Estratégias de marketing verde na percepção de compra dos consumidores na grande São Paulo. In: ENCONTRO DE MARKETING DA ASSOCIAÇÃO NACIONAL DE PÓS-GRADUAÇÃO E PESQUISA EM ADMINISTRAÇÃO, EMA, 3., 2008, Curitiba. Anais... Curitiba: ANPAD, 2008. p. 1-15.

FABRIS, C. Marketing verde nos sites das maiores empresas do país: um estudo com fundamento na "legitimidade" e "isomorfismo". In:

ENCONTRO DE MARKETING DA ASSOCIAÇÃO NACIONAL DE PÓS-GRADUAÇÃO E PESQUISA EM ADMINISTRAÇÃO, EMA, 3., 2008, Curitiba. Anais... Curitiba: ANPAD, 2008. p. 1-14.

FISHBEIN, M.; AJZEN, I. Belief, attitude, intention and behavior: an introduction to theory and research. Mass Reading, MA: Addison Wesley, 1975.

Theory-based behavior change

interventions: comments on Hobbis and Sutton, Journal of Health Psychology, v. 10, n. 1, p. 27-31, 2005. 
FOLLOWS, S. B.; JOBBER, D. Environmentally responsible purchase behavior: a test of a consumer model. European Journal of Marketing, v. 34, n. 5, p. 723-746, 2000.

GARCIA, M. N.; SILVA, D.; PEREIRA, R. S.; ROSSI, G. B.; BAZANINI, R. Percepção sobre a recompensa do consumidor ao comportamento empresarial socioambientalmente responsável. In: ENCONTRO NACIONAL DA ASSOCIAÇÃO NACIONAL DE PÓS-GRADUAÇÃO E PESQUISA EM ADMINISTRAÇÃO, ENANPAD, 32., 2008, Rio de Janeiro, Anais... Rio de Janeiro: ANPAD, 2008. p. 1-15.

GIL, A. P.; CAMARGO, A. S. JR. A influência da preocupação ambiental na segmentação dos consumidores de papel para impressão. In: ENCONTRO DE MARKETING DA ASSOCIAÇÃO NACIONAL DE PÓS-GRADUAÇÃO E PESQUISA EM ADMINISTRAÇÃO, EMA, 3., 2008, Curitiba. Anais... Curitiba: ANPAD, 2008. p. 1-16.

HOEFFLER, S.; KELLER, K. L. Building brand equity through corporate societal marketing. Journal of Public Policy \& Marketing, v. 21, n. 1, p. 78-89, 2002.

JOHANSSON, J. K. Determinants and effects of the use of "made in" labels. International Marketing Review, v. 6, p. 47-58, 1989.

KELLER, K. L. Conceptualizing, measuring, and managing customer-based brand equity. Journal of Marketing, v. 57, p. 1-22, 1993.

KOTLER, P.; KELLER, K. L. Administração de marketing. 12. ed. São Paulo: Prentice Hall, 2006.

KREIDLER, N. B.; JOSEPH-MATHEWS, S. How green should you go? Understanding the role of green atmospherics in service environment evaluations. International Journal of Culture, Tourism and Hospitality Research, v. 3, n. 3, p. 228-245, 2009.

LAGES, N.; NETO, A. V. Mensurando a consciência ecológica do consumidor: um estudo realizado na cidade de Porto Alegre. In: ENCONTRO DA ASSOCIAÇÃO NACIONAL DE PÓS-GRADUAÇÃO E PESQUISA EM
ADMINISTRAÇÃO, ENANPAD, 26., 2002, Salvador, Anais... Salvador: ANPAD, 2002. p. $1-15$.

LIN, S. Exploratory evaluation of potential and current consumers of organic cotton in Hawaii. Asia Pacific Journal of Marketing and Logistics, v. 21, n. 4, p. 489-506, 2009.

LORD, K. R.; LEE, M.; SAUER, P. L. The combined influence hypothesis: central and peripheral antecedents of attitude toward the ad. Journal of Advertising, v. 24, p. 73-86, 1995.

LUTZ, R. J. The role of attitude theory in marketing. In: KASSARJUAN, H. H.; ROBERTSON, T. S. Perspectives in consumer behavior. 4. ed. Englewood Cliffs, NJ: Prentice Hall International Editions, 1991. p. 317-339.

LUTZ, R. J.; MACKENZIE, S. B.; BELCH, G. E. Attitude toward the advertising as a mediator of advertising effectiveness: determinants and consequences. Advances in Consumer Research, v. 10, p. 532-539, 1983.

MACKENZIE, S. B.; LUTZ, R. J.; BELCH, G. E. The role of attitude toward the ad as a mediator of advertising effectiveness: a test of competing explanations. Journal of Marketing Research, v. 23, p. 130-143, 1986.

MALHOTRA, N. K. Pesquisa de marketing: uma orientação aplicada. Porto Alegre: Bookman, 2001.

MINIARD, P. W.; BHATLA, S.; ROSE, R. L. On the formation and relationship of ad and brand attitudes: an experimental and causal analysis. Journal of Marketing Research, v. 27, p. 290303, 1990.

MITCHELL, A. A.; OLSON, J. C. Are product beliefs the only mediator of advertising effects on brand attitude? Journal of Marketing Research, v. 23, p. 318-332, 1981.

MOTTA, S. L. S. Estudo sobre a segmentação de mercado consumidor por atitude $\mathrm{e}$ atributos ecológicos de produtos. 2009, 262 f. Tese (Doutorado em Administração) Departamento de Administração da Faculdade 
de Economia, Administração e Contabilidade Universidade de São Paulo, São Paulo, 2009.

MOWEN, J.; MINOR, M. Consumer behavior. 5. ed. New Jersey: Prentice Hall, 1998.

NEWELL, S. J.; GOLSMITH, R. E.; BANZHAF, E. J. The effect of misleading environmental claims on consumer perceptions of advertisements. Journal of Retailing, p. 48-58, 1998.

OLSON, J. C.; JACOBY, J. Cue utilization in the quality perception process. Advances in Consumer Research, p. 470-475, 1972.

OTTMAN, J. A. Marketing verde: desafios e oportunidades para a nova era do marketing. São Paulo: Makron Books, 1994.

PEDHAZUR, E. J.; SCHMELKIN, L. P. Measurement, design, and analysis: an integrated approach. New Jersey: LEA, 1991.

PEREIRA, S. J. N. O impacto do argumento ecológico nas atitudes dos consumidores: um estudo experimental. 2003, 91 f. Dissertação (Mestrado Executivo em Gestão Empresarial) Escola de Administração Pública e de Empresas Fundação Getúlio Vargas, Rio de Janeiro, 2003.

PETER, J. P.; OLSON, J. C. Consumer behavior and marketing strategy. 4. ed. Chicago: Irwin, 1996.

PICKETT-BAKER, J.; OZAKI, R. Proenvironmental products: marketing influence on consumer purchase decision. Journal of Consumer Marketing, v. 25, n. 5, p. 281-293, 2008.

PIMENTA, M. L.; BOAS, L. H. B. V. Percepções de consumidores de alimentos orgânicos na cidade de Uberlândia na perspectiva de valores: uma aplicação da laddering e cadeia de meios e fins. In: ENCONTRO DE MARKETING DA ASSOCIAÇÃO NACIONAL DE PÓS-GRADUAÇÃO E PESQUISA EM ADMINISTRAÇÃO, EMA, 3., 2008, Curitiba. Anais... Curitiba: ANPAD, 2008. p. 1-16. PORTILHO, F. Consumo verde e cidadania: possibilidades de diálogo? Revista Científica e Cultural da Universidade Estácio de Sá, v.1, n. 3, 1999. Disponível em: < http://www.rubedo.
psc.br/Artigos/consumo.htm>. Acesso em: 17 nov. 2009.

ROBERTS, J. Green consumers in the 1990s: profile and implications for advertising, Journal of Business Research, v. 36, n. 2, p. 217-231, 1996.

ROCHA, A. L. P.; MARQUES, D. L.

S. Marketing verde para quem? In: ENCONTRO DA ASSOCIAÇÃO NACIONAL DE PÓS-GRADUAÇÃO E PESQUISA EM ADMINISTRAÇÃO, ENANPAD, 28., 2004, Porto Alegre. Anais... Porto Alegre: ANPAD, 2004. p. 1-16.

ROMEIRO, M. Um estudo sobre o comportamento do consumidor ambientalmente favorável: uma verificação no ABC paulista. 2006, 358 f.. Tese (Doutorado em Administração) - Departamento de Administração da Faculdade de Economia, Administração e Contabilidade - Universidade de São Paulo, São Paulo, 2006.

SCHIFFMAN, L. G.; KANUK, L. L.

Comportamento do consumidor. Rio de Janeiro: LTC, 2000.

SCHLEGELMILCH, B. B.; BOHLEN, G. M.; DIAMANTOPOULOS, A. The link between purchasing decisions and measures of environmental consciousness. European Journal of Marketing, v. 30, n. 5, p. 35-55, 1996.

SCHULTZ-PEREIRA, J. C.; GUIMARÃES, R. D. Consciência verde: uma avaliação das práticas ambientais. Revista Eletrônica de Gestão, v. 2, n. 1, p. 61-73, 2009.

SHRUM, L. J.; MCCARTY, J. A.; LOWREY, T. M. Buyer characteristics of the green consumer and their implication for advertising strategy. Journal of Advertising, v. 24, n. 2, p. 71-82, 1995.

SOLOMON, M. R. O comportamento do consumidor: comprando, possuindo e sendo. 5 . ed. Porto Alegre: Bookman, 2002.

STRAUGHAN, R. D.; ROBERTS, J. A. Environmental segmentation alternatives: a look at green consumer behavior in the 
new millennium. The Journal of Consumer Marketing, v. 16, n. 6, p. 558-575, 1999.

SUJAN, M. Consumer knowledge: effects on evaluation strategies mediating consumer judgments. Journal of Consumer Research, v. 12, p. 31-45, 1985.

SUJAN, M.; BETTMAN, J. R. The effects of brand positioning strategies on consumers' brand and category perceptions: some insights from schema research, Journal of Marketing Research, v. 16, p. 454-467, 1989.

SZYBILLO, G. J.; JACOBY, J. Intrinsic versus extrinsic cues as determinants of perceived product quality. Journal of Applied Psychology, v. 59, p. 74-78, 1974.

VEIGA, R. T; SANTOS, D. O.; LACERDA, T. $S$. Antecedentes da intenção de consumo de cosméticos ecológicos. In: ENCONTRO DE MARKETING DA ASSOCIAÇÃO NACIONAL DE PÓS-GRADUAÇÃO E PESQUISA EM ADMINISTRAÇÃO, II EMA, 2006, Rio de Janeiro, Anais... Rio de Janeiro: EMA, 2006. p. 1-15. 